\title{
Ballooning instabilities in a Heliotron J plasma
}

$\operatorname{AUTHOR}(S)$ :

Yamagishi, O; Nakamura, Y; Kondo, K

\section{CITATION:}

Yamagishi, O ...[et al]. Ballooning instabilities in a Heliotron J plasma. PHYSICS OF PLASMAS 2001, 8(6): 2750-2759

\section{ISSUE DATE:}

2001-06

URL:

http://hdl.handle.net/2433/50249

\section{RIGHT:}

Copyright 2001 American Institute of Physics. This article may be downloaded for personal use only. Any other use requires prior permission of the author and the American Institute of Physics. 


\title{
Ballooning instabilities in a Heliotron J plasma
}

\author{
Osamu Yamagishi, ${ }^{a}$ Yuji Nakamura, and Katsumi Kondo \\ Graduate School of Energy Science, Kyoto University, Uji, Kyoto 611-0011, Japan
}

(Received 5 January 2001; accepted 28 February 2001)

\begin{abstract}
Ideal magnetohydrodynamic stability analysis of local pressure-driven modes in an $L=1$ heliotron, Heliotron J [M. Wakatani et al., Nucl. Fusion 40, 569 (2000)], is investigated by means of three-dimensional (3D) ballooning formalism and the Mercier criterion. In 3D systems such as heliotrons, the ballooning modes are separated into two categories: One is tokamak-like ballooning modes which are localized only in the poloidal direction, and the other is modes inherent to 3D systems which are localized on the specific flux tubes. The tokamak-like ballooning modes change to the Mercier modes in the limit that the mode is sufficiently extended along the field line, but the nonaxisymmetric ballooning mode does not so. The $L=1$ Heliotron J equilibrium investigated here has weak global shear and the dominant Fourier amplitudes of magnetic-field strength is rather different from the conventional helical systems with $L=2$ helical coils. Since the weak global shear causes the reduction of integrated local shear along the field lines easily, which combines with strongly modulated destabilizing effects on the flux surface, the nonaxisymmetric ballooning modes localized on the specific flux tubes can become unstable. On the other hand, the Mercier modes are suppressed due to the deep magnetic well. The results obtained from the model equilibrium of $L$ $=2$ Large Helical Device (LHD), for which several reports have already published [N. Nakajima, Phys. Plasmas 3, 4556 (1996), for example], are also shown and compared with the results of Heliotron J. The LHD equilibrium employed here has a magnetic hill region at the outer radius, and this tends to make the Mercier modes unstable. It is found that this difference of the Mercier stability property in two equilibria is concerned in the local ballooning stability, and the notable difference of local dispersion relations appears. It is also found from the comparison of two systems that the nonaxisymmetric ballooning modes have a similar property to the tokamak-like ballooning modes, in the sense of the $\bar{s}-\bar{\alpha}$ diagram where $\bar{s}$ and $\bar{\alpha}$ are shear and pressure gradient parameter. (C) 2001 American Institute of Physics. [DOI: 10.1063/1.1367321]
\end{abstract}

\section{INTRODUCTION}

Ideal magnetohydrodynamic (MHD) pressure-driven instabilities in finite- $\beta$ magnetically confined plasmas are dangerous and ought to be suppressed. The high- $n$ ( $n$ is a toroidal mode number) pressure-driven modes have flute-like structures due to the tension parallel to field lines confining a plasma. As is well known in the tokamak literature, the modes are classified into the interchange-like and ballooning-like modes by whether the mode spreads over a flux surface or localizes in the bad curvature region usually formed in the outside of the torus. The stability of the interchange modes in the limit of long parallel wavelength can be determined by the Mercier criterion in terms of the flux surface quantities. On the other hand, the stability analysis of high- $n$ ballooning modes requires the local quantities as well as flux surface quantities. Within the framework of the threedimensional (3D) ballooning formalism, ${ }^{1}$ it was found that in 3D systems such as heliotrons, the above classification on the structure of the pressure-driven modes in tokamaks is inadequate, since the ballooning feature characterized by the localization stems essentially from modulations of the equi-

${ }^{a)}$ Electronic mail: yamagisi@center.iae.kyoto-u.ac.jp librium magnetic structure. That is, the modes inherent in 3D systems with another degree of freedom on the localization potentially appear.

Recently several local stability analyses of ballooning modes in a heliotron plasma have been reported. ${ }^{2-4}$ A Large Helical Device (LHD) equilibrium, which is given in Ref. 5, has been chosen as a model equilibrium in the above papers. LHD is an $L=2 / M=10$ planar axis heliotron, where $L$ is the pole number of helical coil winding and $M$ is the number of toroidal periods. The LHD equilibrium has strong global shear and a large helical component of magnetic-field spectra compared with other components. The local dispersion relation $\lambda\left(s, \theta_{k}, \alpha\right)=\omega^{2}$ of LHD has been evaluated by solving the ballooning mode equation in the parameter space $\left(s, \theta_{k}, \alpha\right)$. Here $s$ is a flux surface label, $\theta_{k}$ is a radial wave number, and $\alpha$ is a field line label, which is an ignorable coordinate in a tokamak. [On the definition of $\left(s, \theta_{k}, \alpha\right)$, see also Sec. III.] It was found in Refs. 2-4 that in the Mercier unstable LHD equilibrium (i.e., with "broad pressure profile" in Ref. 5), $\lambda=$ const. isosurfaces show two topologically different structures in the parameter space $\left(s, \theta_{k}, \alpha\right)$. One shows a cylindrical structure whose axis is in the $\alpha$ direction in the parameter space, reflecting a moderate $\theta_{k}$ and weak $\alpha$ dependence. These are topologically the same as those in the axisymmetric system where no $\alpha$ dependence exists. The coherent structure of cylindrical surfaces in the $\alpha$ 
direction enable one to extrapolate from infinite- $n$ results to finite- $n$ by requiring the periodicity of the global mode in the toroidal direction (what is called the "quantization condition" ${ }^{\prime}$ ). These modes on cylindrical surfaces are, in general, considered as tokamak-like ballooning modes or interchange modes because of their weak $\alpha$ dependence. They were called the "interchange branch" in Ref. 4. The global eigenvalues of low- $n$ modes were evaluated by applying the quantization condition to this branch. ${ }^{3,4}$ The other is inherent in 3D systems, where the isosurfaces of eigenvalue form the isolated spherical surfaces in the parameter space, reflecting a strong $\alpha$ dependence as well as a $\theta_{k}$ dependence. They were called the "ballooning branch." 4 In addition to the $\theta_{k}$ dependence, which indicates the well-known poloidal localization, the $\alpha$ dependence indicates the localization of the modes on the specific flux tubes in 3D systems. It was predicted from this reason that the $\left(s, \theta_{k}, \alpha\right)$ dependence of the local dispersion relation indicates the localization of the global mode in the radial, poloidal, and toroidal direction, respectively. ${ }^{2}$ This has been confirmed by performing the global analysis. ${ }^{6}$

In contrast with such an $L=2$ heliotron, Heliotron J, which is an $L=1 / M=4$ helical axis heliotron, has relatively weak global shear in a standard configuration. The magnetic structure is strongly modulated due to the interplay among the helicity common to heliotrons, the toroidicity common to tori, and the large bumpiness. Here "bumpiness" denotes the degree of the modulation of magnetic field strength in the toroidal direction, and is also called the toroidal mirror ratio. ${ }^{7}$ Since the competition between the stabilizing local shear and the driving source reflecting the local magnetic structure is essential for the stability of the pressure-driven modes, it is important to investigate the stability property in this low shear heliotron. For this purpose, we have studied the local ballooning and Mercier stability in an $L=1$ Heliotron $\mathbf{J}$ plasma. The role of shear and magnetic structure on the local stability is discussed. In particular, it is found that the bumpiness plays an important role. We also show the results from the LHD equilibrium. The above discussion for the two branches in an LHD equilibrium will be revisited. It will be shown that the modes belonging to the "interchange branch" in Ref. 4 correspond to the interchange modes in the LHD equilibrium employed here. The results obtained from the LHD equilibrium are compared with those of Heliotron J.

The equilibrium configuration of Heliotron $\mathrm{J}$ in this study is characterized by a deep magnetic well and weak global shear. As shown in the following section, the Mercier criterion predicts that the Mercier modes are stable in the typical equilibrium of Heliotron $\mathrm{J}$ up to the equilibrium beta limit in favor of the deep magnetic well, or favorable surface-averaged magnetic curvature. However, it is found that the nonaxisymmetric ballooning modes, which are localized on the specific flux tubes, can become unstable at low beta. In the case of the model LHD equilibrium employed here, such nonaxisymmetric ballooning modes are suppressed in favor of the large global shear and the interchange-like modes can become unstable precedently as beta increases. These will be related to the difference of the local dispersion relation in Heliotron $\mathrm{J}$ and LHD.

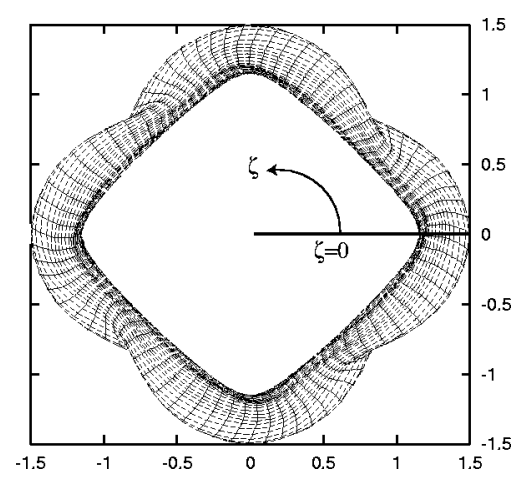

FIG. 1. Top view of a Heliotron-J plasma.

In Sec. II, MHD equilibrium of Heliotron J and associated equilibrium quantities are represented in some detail. The result from the Mercier criterion is also shown in this section. In Sec. III, the ballooning mode equation and its solution method are briefly described. Sec. IV is devoted to the results for an LHD equilibrium (Sec. IV A) and for a Heliotron J equilibrium (Sec. IV B). Conclusions are given in Sec. V.

\section{MHD EQUILIBRIUM OF A HELIOTRON J PLASMA}

The equilibrium configuration of an $L=1 / M=4$ helical axis heliotron device, Heliotron $\mathrm{J}^{7}{ }^{7}$ is described here. The MHD equilibrium is calculated by the VMEC code, ${ }^{8}$ in which the number of magnetic surfaces are 101, and the poloidal and toroidal components in the Fourier representation, $0 \leqslant m \leqslant 11$ and $-12 \leqslant n / M \leqslant 12$, respectively, are used. The outermost flux surface required as a fixed boundary constraint is obtained from the field line tracing in vacuum, which is done by the KMAG code. ${ }^{9}$ In this study, we choose a vacuum configuration for the basic one in experiments. The net toroidal current is prescribed to be zero and pressure profile is prescribed as noted later. After computing the equilibrium in VMEC coordinates, the evaluated magnetic field is mapped into Boozer coordinates ${ }^{10}$ for analytical and numerical tractability in the stability analyses. NEWBOZ code is employed to this end, in which the poloidal and toroidal Fourier components, $0 \leqslant m \leqslant 23$ and $-10 \leqslant n / M \leqslant 10$ are used. (On the coordinate transformation in NEWBOZ code, see, e.g., Appendix B in Ref. 11.)

In Fig. 1, the top view of a Heliotron J plasma is shown. In our equilibrium calculation, the origin of the poloidal angle $\theta$ corresponds to the outside of torus. The origin of the toroidal angle $\zeta$ is chosen as the point where the helical coil passes inside the torus. The plasma is expanded to the outside from the major axis and the shape of the poloidal cross section is elongated horizontally at $M \zeta=0$. The outboard of the plasma at $M \zeta=\pi$ is located just inside the helical coil and a corresponding poloidal cross section is elongated vertically. Figure 2 shows the radial dependence of the dominant Fourier harmonic amplitudes of magnetic-field strength, $B_{m n}$ normalized by $B_{00}$ at the plasma edge, at $\beta_{0}=0.0 \%$. Here $s$ is the toroidal flux normalized to unity at the plasma edge and $\beta_{0}$ is a central beta value, $\beta_{0}=2 p(0) / B_{0}^{2}$, with $p(0)$ being the pressure at the magnetic axis and $B_{0}$ being 


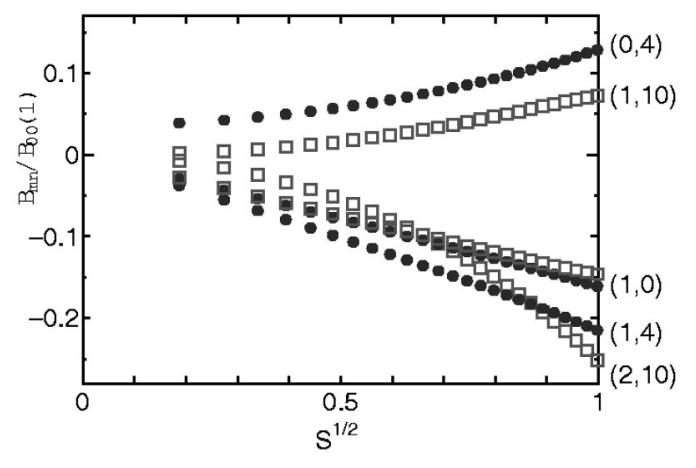

FIG. 2. Radial dependence of $B_{m n}$ normalized by $B_{00}$ at plasma edge, as a function of $s^{1 / 2}$. Not only Heliotron-J (circles) but also LHD (squares) case at $\beta_{0}=0.0 \%$ are shown for comparison.

the toroidally averaged strength of vacuum magnetic field at $R=R_{0}(=1.2 \mathrm{~m}$ for Heliotron $\mathrm{J})$. The case for LHD with the same central beta is also shown for comparison. It is found in both systems that the helical components $(L, M)$ are the largest and the toroidal components $(1,0)$ are comparable, but in Heliotron $\mathrm{J}$, the bumpy component $(0, M)$ is very large. This bumpy field is characteristic of Heliotron $\mathrm{J}$ and can be controlled widely with toroidal coil currents. ${ }^{7}$

In Fig. 3, the radial dependence of typical equilibrium quantities; (a) safety factor, (b) global magnetic shear, (c) magnetic well depth, and (d) Mercier coefficient are shown for several beta equilibria with the broad pressure profile, $p$ $=p(0)\left(1-s^{2}\right)^{2}$. Here the global magnetic shear $s_{*}$ is defined in Eq. (8) in Sec. III and the well depth is defined as $-\left[V^{\prime}(s)-V^{\prime}(0)\right] / V^{\prime}(0)[\%]$ with the volume $2 \pi V(s)$ within the magnetic surface labeled by $s$. Positive (negative) derivative of the well depth curve with respect to surface label $s$ denotes the magnetic well (hill) at $s$. The Mercier coefficient $D_{M}(s)>0$ denotes that the system is stable for
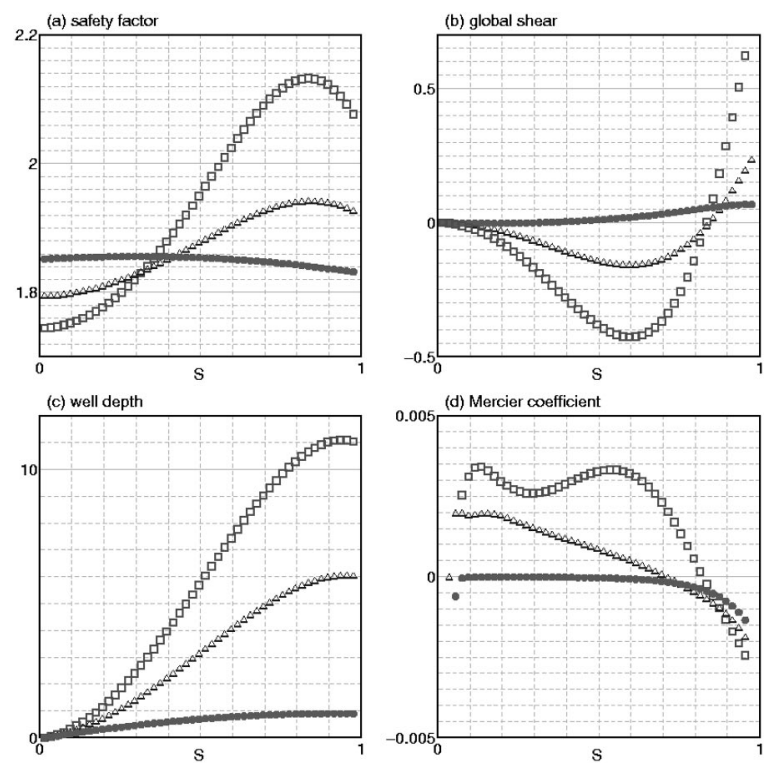

FIG. 3. Radial dependence of the equilibrium quantities for the broad pressure profile, (a) safety factor, (b) global shear, (c) well depth, (d) Mercier coefficient with central beta value $\beta_{0}=0.0 \%$ (circles), $\beta_{0}=1.0 \%$ (triangles), $\beta_{0}=2.0 \%$ (squares), as a function of $s$.
Mercier modes. It is found from Figs. 3(a) and 3(b) that the safety factor is nearly constant and the shear is very weak at $\beta_{0}=0.0 \%$. As beta increases, the magnitude of global shear becomes larger. The shear becomes more negative from the axis to half radius due to the Pfirsh-Schlüter current and more positive toward plasma edge due to the poloidal field by the helical coil current, so there exists a zero shear point between the negative and positive shear regions. However, the magnitude of global shear is still small in this equilibrium. Figure 3(c) shows that the magnetic well is formed in almost the entire region and the increase of beta deepens it further. An LHD equilibrium with a broad pressure profile, for example, tends to be Mercier unstable because its steep pressure gradient region overlaps the magnetic hill region usually formed in the outer radius. Thus, the radially broad region of the magnetic well in Heliotron $\mathrm{J}$ is expected to be favorable against the interchange modes, even in the equilibrium with a broad pressure profile. The Mercier criterion shown in Fig. 3(d) is determined by these surface (and surface-averaged) quantities, and the Mercier modes become stable in almost all regions due mainly to the deep magnetic well, although the global shear is weak. It is noted that the Mercier unstable region exists near the plasma edge at zero beta, as well as finite beta. The Mercier coefficient at zero beta ought to include only the shear stabilizing effect, so that the presence of a Mercier unstable region at zero beta indicates that the accuracy of the equilibrium calculation in those regions is not sufficient for stability analysis and we should exclude the results there.

As is mentioned above, the magnetic well plays an important role in the Mercier stability. This is understood in terms of the distribution of magnetic curvature on each surface. In Fig. 4(a), the normal magnetic curvature given in Eq. (4) in Sec. III with minus sign, $-\kappa_{n}$ is shown in one field period for Heliotron J. The negative, concave regions show locally good curvature and positive, convex regions show locally bad curvature. The area of bad curvature due to the toroidicity (the outside of torus) and the helicity (opposite side of helical coil) is smaller than the good curvature area. Therefore, the surface-averaged curvature is negative, implying a magnetic well. The magnetic well is mainly determined by the vacuum configuration, and it is expected that the increase of the bumpiness tends to make the magnetic well deep. ${ }^{7}$ Also as beta increases, the deformation of the surface shape causes the change of distribution of the normal curvature and the magnetic well becomes deeper, as shown in Fig. 4(a). This is a typical feature of Heliotron J equilibrium and leads the Mercier modes to be stable up to the equilibrium beta limit, even if the pressure profile is broad.

\section{THE BALLOONING MODE EQUATION}

In order to reduce the full $3 \mathrm{D}$ problem to a $1 \mathrm{D}$ equation governing the local ballooning modes, the WKB (WentzelKramers-Brillouin) (eikonal) approximation is applied to the perturbed displacement vector $\boldsymbol{\xi}(\mathbf{r})=\hat{\boldsymbol{\xi}}(\mathbf{r}) \exp [i S(\mathbf{r})]$ in the MHD Laglangian $\mathcal{L}=\omega^{2} \mathcal{K}-\delta W .{ }^{1}$ Here the temporal dependence of the perturbed displacement vector is assumed as $\boldsymbol{\xi}$ $\sim \exp (-i \omega t)$, where $\omega^{2}$ is a purely real number by virtue of 
(a)

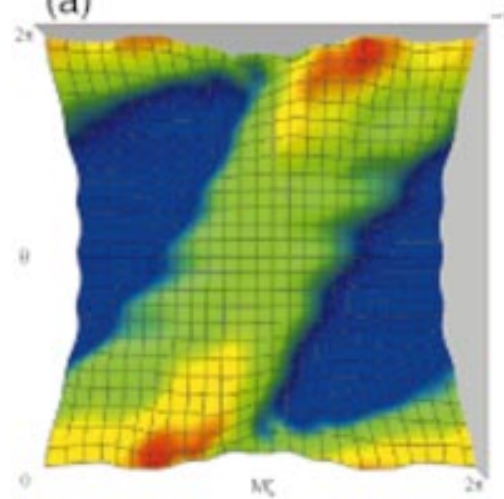

(b)
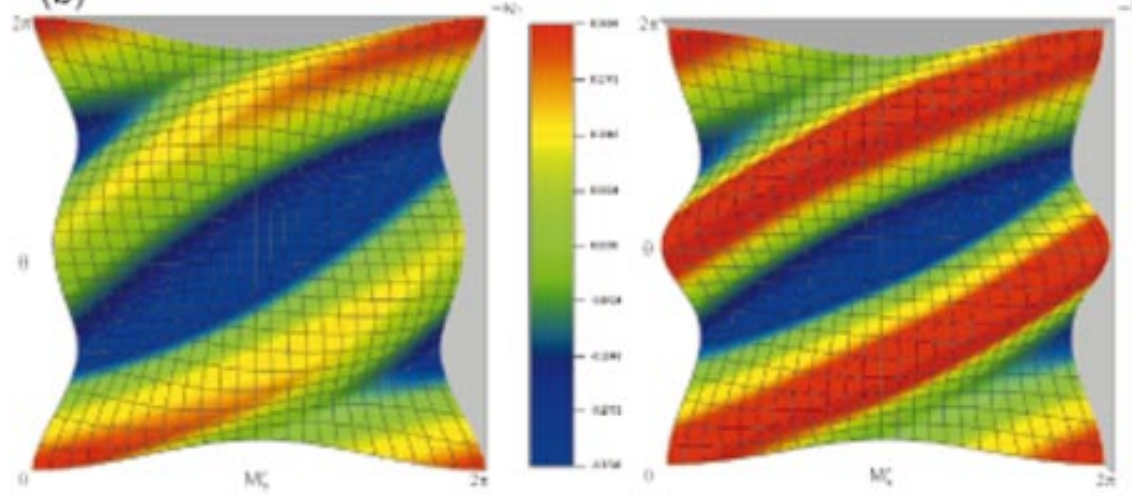
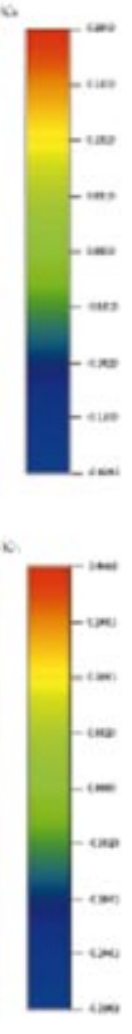

FIG. 4. (Color) Normal curvature in one field period. Positive (convex) and negative (concave) regions correspond to good and bad curvature regions, respectively. (a) The case of Heliotron J equilibrium for the broad pressure profile at $\beta_{0}=0.0 \%$ (left) and $\beta_{0}=2.0 \%$ (right) at $s=0.75$. (b) The case of LHD equilibrium for the broad pressure profile at $\beta_{0}=2.0 \%$ at $s=0.25$ (left) and $s=0.75$ (right). the self-adjointness of the force operator in ideal MHD. Then the usual assumption is made that the mode structure is flutelike, i.e., its perpendicular wavelength is sufficiently short compared with the parallel wavelength. The eikonal $S$ then describes the rapid variation of modes perpendicular to the field line, assuming the lowest order wave number vector $\mathbf{k}$ $=\nabla S$ to lie in the perpendicular direction; $\mathbf{B} \cdot \nabla S=0$, while $\hat{\boldsymbol{\xi}}$ is considered as slowly varying envelope. According to the usual procedure to extremize $\mathcal{L}$, the resulting incompressible balloning mode equation in Boozer coordinates is given as ${ }^{1,4}$

$$
\frac{d}{d \theta}\left[\mathcal{A} \frac{d \xi}{d \theta}\right]+\left[\mathcal{K}+\frac{\mathcal{A}}{B^{\theta^{2}}} \rho_{m} \omega^{2}\right] \xi=0
$$

where

$$
\begin{aligned}
& \mathcal{A}=\chi^{\prime 2} \frac{B^{\theta}}{B^{2}}\left|\frac{\mathbf{k}}{k_{\alpha}}\right|^{2}, \\
& \mathcal{K}=\frac{2 \mu_{0} p^{\prime}}{B^{\theta}}\left[\kappa_{n}+q^{\prime}\left(\theta-\theta_{k}\right) \kappa_{g}\right], \\
& \boldsymbol{\kappa}_{n} \equiv \boldsymbol{\kappa} \cdot e_{s}=\frac{1}{B} \frac{\partial B}{\partial s}+\frac{\mu_{0} p^{\prime}}{B^{2}}-B_{s} \frac{B^{\theta}}{B^{3}} \frac{d B}{d \theta}, \\
& \kappa_{g} \equiv \boldsymbol{\kappa} \cdot \mathbf{g}=\frac{B^{\theta}}{B^{3}}\left(I \frac{\partial B}{\partial \zeta}-G \frac{\partial B}{\partial \theta}\right), \\
& \mathbf{e}_{s}=\sqrt{g} \nabla \theta \times \nabla \zeta, \quad g=\nabla \zeta-q \nabla \theta, \\
& \left|\frac{\mathbf{k}}{k_{\alpha}}\right|^{2}=\left(\frac{q \psi^{\prime}}{2 \psi}\right)^{2}|\nabla s|^{2}\left[\int_{\theta_{k}} \hat{s}_{*} d \theta\right]^{2}+\frac{B^{2}}{|\nabla \chi|^{2}},
\end{aligned}
$$

$$
\begin{aligned}
& \hat{s}_{*} \equiv \frac{2 \psi}{q \psi^{\prime}} \frac{d}{d \theta}\left(\frac{\nabla \alpha \cdot \nabla s}{|\nabla s|^{2}}\right)=s_{*}+\widetilde{s}_{*}, \\
& s_{*}=2 \frac{d \ln t}{d \ln \psi}, \quad \widetilde{s}_{*}=\frac{2 \psi}{q \psi^{\prime}} \frac{d}{d \theta}\left(\frac{\mathbf{g} \cdot \nabla s}{|\nabla s|^{2}}\right),
\end{aligned}
$$

$s=\psi / \psi_{\text {edge }}$ is the toroidal flux normalized by its edge value, $2 \pi \chi$ is the poloidal flux inside the magnetic surface $s, q$ is the safety factor, $\ell=1 / q$ is the rotational transform, $2 \pi I / \mu_{0}$ and $2 \pi G / \mu_{0}$ are the toroidal current inside and the poloidal current outside the surface $s$, respectively, $\mu_{0}$ is permeability in vacuum, and $\rho_{m}$ is mass density, which is assumed to be unity. The field line label, $\alpha=\zeta-q \theta$, is an ignorable coordinate in axisymmetric systems, $\zeta$ is the toroidal angle and $\theta$ is the extended poloidal angle defined in the covering space $(-\infty<\theta<\infty) .{ }^{1}$ The wave number vector with two degrees of freedom is defined as $\mathbf{k}=\nabla S=k_{\alpha} \nabla \alpha+k_{q} \nabla q$ with $k_{\alpha}$ $=\partial S / \partial \alpha$ and $k_{q}=\partial S / \partial q . \theta_{k}=k_{q} / k_{\alpha}$ is the radial wave number and plays the role of the origin of eikonal phase. $\kappa_{n}$ and $\kappa_{g}$ are the normal and geodesic components of the curvature vector $\boldsymbol{\kappa}=B^{-2} \nabla \cdot\left(\mid-\mathbf{B B} / B^{2}\right)\left(\mu_{0} p+B^{2} / 2\right)$ with $\mid$ the unit dyadic. The magnetic field is expressed as $\mathbf{B}=\nabla \alpha \times \nabla \chi$, and $B^{\theta}=\mathbf{B} \cdot \nabla \theta$ and $B_{s}=\mathbf{B} \cdot \mathbf{e}_{s}$ are the contravariant poloidal component and covariant radial component of the magnetic field, respectively, and $\sqrt{g}=(\nabla s \cdot \nabla \theta \times \nabla \zeta)^{-1}$ is the Jacobian of transformation from the real space. The local shear $\hat{s}_{*}=s_{*}$ $+\widetilde{s}_{*}$ is the sum of the global shear $s_{*}$ and the oscillating shear $\widetilde{s}_{*}$. Prime denotes the derivative with respect to the surface label $s$ and $d / d \theta=\left(B^{\theta}\right)^{-1} \mathbf{B} \cdot \nabla$ is the derivative 
along the field line. $\omega^{2}$ and $\xi$ is the eigenvalue and the corresponding eigenfunction along the field line.

The term $\mathcal{A}$ in Eq. (1), which is proportional to $\left|\mathbf{k} / k_{\alpha}\right|^{2}$ by noting that $B^{\theta} / B^{2}$ is a surface quantity in Boozer coordinates, represents the stabilizing tension arising from bending the field line, and the second term $\mathcal{K}$ proportional to $p^{\prime}$ is destabilizing (stabilizing) pressure-driven term if it is positive (negative). We can solve it as an ordinary differential eigenvalue equation along the field line with three input parameters $\left(s, \theta_{k}, \alpha\right)$. The end points of $\theta$ on covering space in numerical procedure, $\pm \theta_{\max }$ are chosen as $\theta_{\max }=20 \pi$ unless otherwise remarked. The sign of eigenvalue $\omega^{2}$ determines the local stability of the system.

In the above ballooning formalism, $q^{\prime} \neq 0$ is assumed to assure an angle-like behavior of $\theta_{k}$, which is needed to construct periodic solutions, ${ }^{1}$ so that the ballooning mode equation cannot be applied in the shearless regime. It is also noted that a stable eigenvalue depends on the the given boundaries $\pm \theta_{\max }$, since it is conjectured that most of the stable eigenvalues are in a continuum. ${ }^{12}$ In addition, $\kappa^{n}$ $=\boldsymbol{\kappa} \cdot \nabla s /|\nabla s|$ and $\kappa_{g}=\boldsymbol{\kappa} \cdot \nabla s \times \mathbf{B} /(|\nabla s| B)$ are often used as the definitions of normal and geodesic curvature. We prefer to use Eqs. (4) and (5) because of their simplicities of representation, but both are basically cousins and, of course, yield the completely equivalent product, Eq. (1). The shooting method is used to solve the eigenvalue problem, Eq. (1), from both the end points $\theta= \pm \theta_{\max }$ with the boundary conditions, $\xi\left( \pm \theta_{\max }\right)=0$, to the midpoint $\theta=0$ with the matching condition, $\quad d \ln \xi /\left.d \theta\right|_{\theta=+0}=d \ln \xi /\left.d \theta\right|_{\theta=-0}$. The fourth-order Runge-Kutta method is used to integrate and numerical accuracy is checked with the more accurate Adams method. ${ }^{13}$ The evaluated eigenvalues $\omega^{2}$ are normalized by the Alfvén frequency, $\omega_{A}^{2}=\left[B_{0} /\left(\rho_{m}^{1 / 2} R_{0}\right)\right]^{2}$ with $\rho_{m}=1$, i.e., hereafter the eigenvalues shown is $\omega_{n}^{2}=\omega^{2} / \omega_{A}^{2}$.

\section{RESULTS}

\section{A. Ballooning mode analysis in LHD}

LHD is an $L=2 / M=10$ planar axis heliotron. The model equilibrium employed here has the vacuum magnetic axis shifted $15 \mathrm{~cm}$ inward from the center of helical coil winding by adjusting the poloidal coil currents. ${ }^{5}$ A broad pressure profile $p=p(0)\left(1-s^{2}\right)^{2}$ and no net current are prescribed as well as Refs. 2-4. We choose the origin of $(\theta, \zeta)$ to locate on the outside of the horizontally elongated poloidal cross section as well as Heliotron J. [Note that in above papers using the LHD equilibrium, the origin of $(\theta, \zeta)$ was on the outside of the vertically elongated poloidal cross section.] The equilibrium quantities are shown in Refs. 2 and 6 and there is magnetic hill region in the outer radius and a substantial stellarator shear $\left(q^{\prime}<0\right)$. As already noted, the combination of the broad pressure profile and the magnetic hill in the edge region of this inward-sifted plasma tends to make the Mercier modes unstable and as expected, the Mercier unstable region appears over $\beta_{0} \sim 1.5 \%$ in the magnetic hill region. Here the normal curvature of LHD equilibrium at $s=0.25$ and 0.75 are shown in Fig. 4(b) for $\beta_{0}=2.0 \%$. The bad curvature region occupies a broader area in one field period at $s=0.75$ and this yields the magnetic hill. It is noted that
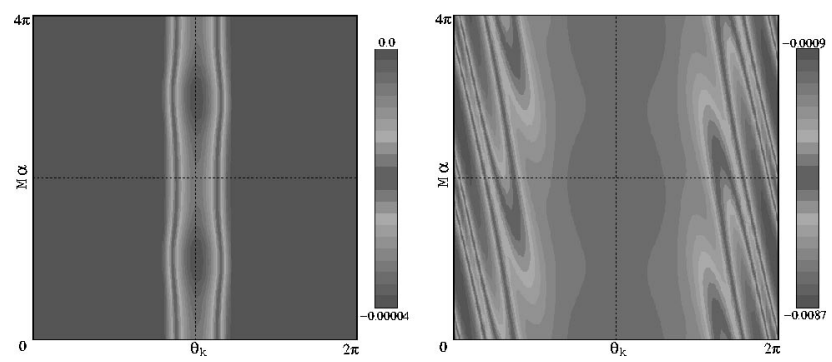

FIG. 5. Contours of the eigenvalues at $s=0.55$ in LHD equilibrium for the broad pressure profile. The $\beta_{0}=2.0 \%$ (left) and $\beta_{0}=4.0 \%$ (right) cases are shown.

the amplitude of the unfavorable curvature is comparable for the inside and outside of the torus at $s=0.75$. This is due to the large helical component in the $L=2$ LHD equilibrium.

Figure 5 shows the contours of eigenvalues on the $\theta_{k}$ $-\alpha$ plane at fixed $s(=0.55)$ for $\beta_{0}=2.0 \%$ and $4.0 \%$ cases, where stable eigenvalue is taken to be zero. It is found that the modes with weak $\alpha$ and moderate $\theta_{k}$ dependence arise near $\theta_{k} \sim \pi$ for the $\beta_{0}=2.0 \%$ case. This is the "interchange branch" in Ref. 4 . When beta increases up to $4 \%$, the interchange branch still exists, extending its domain in the parameter space, while isosurfaces with the strong $\alpha$ and $\theta_{k}$ dependence appear near $\theta_{k} \sim 2 m \pi$ with any integer $m$, which is the "ballooning branch." In order to see the relation between the "interchange branch" and the Mercier modes, the radial positions of the marginal stability boundary from the Mercier criterion and the ballooning mode equation are shown in Fig. 6. Here the dash-dotted line shows the Mercier stability boundary and the dashed and solid line show the stability boundaries of the ballooning results for $\theta_{\max }=20 \pi$ and $1000 \pi$, respectively. For the ballooning results, $\left(\theta_{k}, M \alpha\right)$ $=(\pi, 0)$ is taken, which corresponds to the center of the interchange branch. The results for the $\theta_{\max }=1000 \pi$ case yields more a severe condition than that for $\theta_{\max }=20 \pi$ for stability. This is because the mode near the marginal stability is quite extended along the field line, so that the case for $\theta_{\max }=20 \pi$ fails to take the mode extent into account correctly. It is confirmed that the radial marginal points for the ballooning results approach the Mercier marginal boundaries

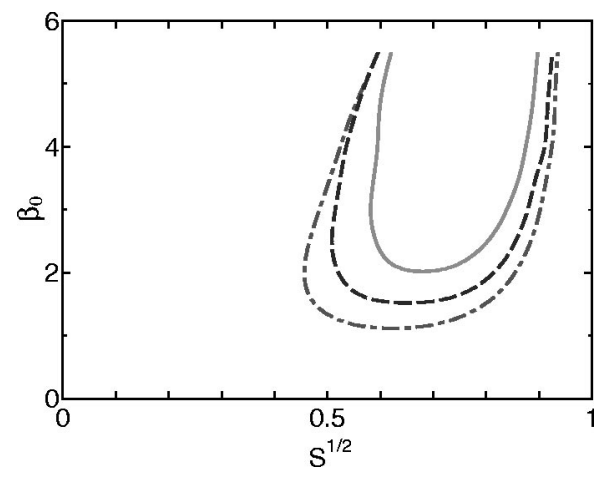

FIG. 6. Stability boundary diagram on $s-\beta_{0}$ plane obtained from the results of the Mercier criterion (dash-dotted line) and the ballooning mode equation with $\theta_{\max }=20 \pi$ (solid line) and $\theta_{\max }=1000 \pi$ (dashed line). The mode belonging to the interchange branch is unstable inside the Mercier boundary. $M \alpha=0$ and $\theta_{k}=\pi$ are taken for ballooning results. 

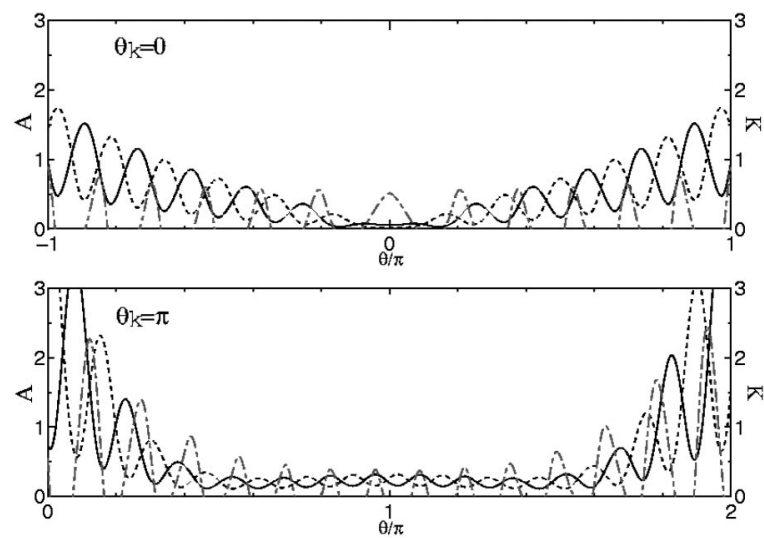

FIG. 7. Stabilizing term $\mathcal{A}$ with $M \alpha=0$ (solid lines) and $M \alpha=\pi$ (dashed lines) and the destabilizing term $\mathcal{K}(>0)$ (dash-dotted lines) with $M \alpha=0$, for $\left(s, \theta_{k}\right)=(0.66,0)$ (upper) and $\left(s, \theta_{k}\right)=(0.52, \pi)$ (lower) cases for the broad pressure profile equilibrium at $\beta_{0}=4.0 \%$.

as $\theta_{\max }$ increases. In this strongly Mercier unstable equilibrium, as a matter of fact, the radial marginal boundaries for the ballooning branch, which correspond to taking $\theta_{k}=0$, are comparable with that for the interchange branch. However, the modes of ballooning branch can be unstable beyond the Mercier unstable region, and we confirmed this by using the equilibrium with a peaked pressure profile, which is more stable for the Mercier modes. Therefore, the circumference of the interchange branch in the parameter space is formed by the interchange modes in the high- $n$ limit, i.e., the Mercier modes.

In order to see the reason why the separate branches appear, the stabilizing effect $\mathcal{A}$ in Eq. (2) with $M \alpha=0$ (solid lines) and $M \alpha=\pi$ (dashed lines), and destabilizing effect, i.e., only positive part of $\mathcal{K}$ (dash-dotted lines) in Eq. (3) with $M \alpha=0$ are shown in Fig. 7 for $\theta_{k}=0$ (upper figure) and $\theta_{k}=\pi$ (lower figure). It is found that changing the field line label $M \alpha$ diametrically yields the local phase shift in $\mathcal{A}$ along the field line. On the other hand, it can be seen that the diametric $\theta_{k}$ parameters yield the change of envelope of $\mathcal{A}$. (Although the $\mathcal{K}$ with $M \alpha=\pi$ is not shown for simplicity, it has inverse phase to $M \alpha=0$ case as well as the $\mathcal{A}$.) From these envelopes of stabilizing $\mathcal{A}$, it is expected that the mode with $\theta_{k}=0$ is extended within $\left|\theta-\theta_{k}\right| \leqq 0.2 \pi$ along the field line, while in the case with $\theta_{k}=\pi$, the mode is extended within $\left|\theta-\theta_{k}\right| \leq \pi$. Hence in the case of $\theta_{k}=\pi$, the local phase shift by changing $M \alpha$ is less important than in the case of $\theta_{k}=0$, and the modes destabilized by several helical ripples of $\mathcal{K}$ in the inside of torus will become insensitive on the $M \alpha$ label. For the $\theta_{k}=0$ case, in contrast, the local phase affects the modes directly as might be seen in upper figure in Fig. 7, and causes the strong $\alpha$ dependence of the modes. Such an envelope of the $\mathcal{A}$ is ascribable mainly to a measure of flux surface interval, $|\nabla s|^{2}$. In the case of $\theta_{k}=0$, which corresponds to the local modes on the outboard of the torus, the $\mathcal{A}$ can rapidly increase along the field line due to the narrow surface interval or large $|\nabla s|^{2}$. Whereas in the case of $\theta_{k}=\pi$, which corresponds to the modes on the inboard of the torus, the $\mathcal{A}$ is slow to increase due to the small $|\nabla s|^{2}$.

If we see the central region of the cylindrical surfaces in

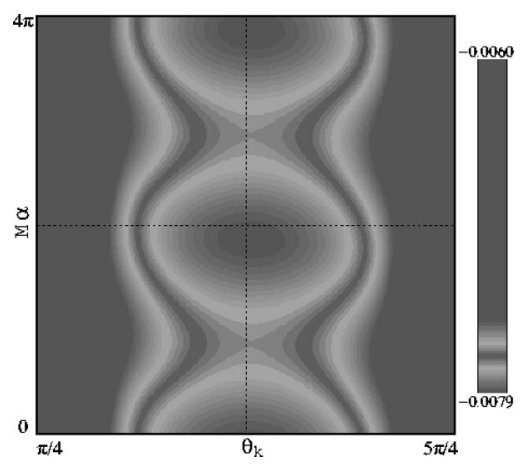

FIG. 8. Contour of the eigenvalues in the central region of the interchange branch at $s=0.55$ for $\beta_{0}=4.0 \%$ LHD equilibrium.

detail, as shown in Fig. 8, there exist spherical isosurfaces, although the parameter dependence is more moderate than that of ballooning branch. This is reasonable, since the modes on this spherical isosurfaces are still locally driven by the bad curvature in the inside of horizontally elongated cross section. As shown in Fig. 4(b), the destabilizing effects $\mathcal{K}$ is comparable in both the inside and outside of the torus in the LHD equilibrium considered here. On the other hand, the Shafranov shift at finite beta causes the notable difference of the stabilizing effects $\mathcal{A}$ between the inside and outside of the torus, as shown in Fig. 7. It follows that the $\alpha$ dependence of the modes is stronger (weaker) in the outside (inside) of the torus as beta increases. These are the "ballooning branch" and "interchange branch," respectively.

As for the ballooning branch in LHD, the modes with strong dependence on $\alpha$ and $\theta_{k}$ cannot arise up to relatively high beta in favor of the large global shear. In such a system, a contribution in the stabilizing $\mathcal{A}$ term or integrated local shear is dominated by a global shear part at low beta, as shown in the next subsection. The reduction of the integrated local shear is weak at least in a low beta plasma. It is considered that the nonaxisymmetric ballooning mode in LHD has similar property to that in the first stability regime of the well-known " $\bar{s}-\bar{\alpha}$ diagram" in tokamak, where $\bar{s}$ and $\bar{\alpha}$ is the shear and pressure gradient parameter.

By making reference to Chen et al. ${ }^{6}$ where our equilibrium corresponds to their "helicity-dominant Mercierunstable equilibrium," it can be seen the relation between the local modes and the global modes. As noted above, for the local modes on a cylindrical isosurface with weak $\alpha$ dependence, the eigenvalue of low- $n$ global mode can be obtained by the quantization condition with a specific toroidal mode number $n$. In the global analysis, the global mode corresponding to the interchange branch can be obtained not only by $3 \mathrm{D}$ global stability codes but also by $2 \mathrm{D}$ codes based on the stellarator approximation. ${ }^{5}$ On the other hand, the quantization for a specific $n$ fails for the local modes with strong $\alpha$ dependence on a spherical surface. The corresponding global mode cannot be obtained by the stellarator approximation. Since the stellarator approximation requires the assumption of the weak toroidal mode coupling, it is suspected that the strong $\alpha$ dependence of the local eigenmodes corresponds to the strong toroidal mode coupling in the global modes. Indeed the 3D global code shows that the mode 

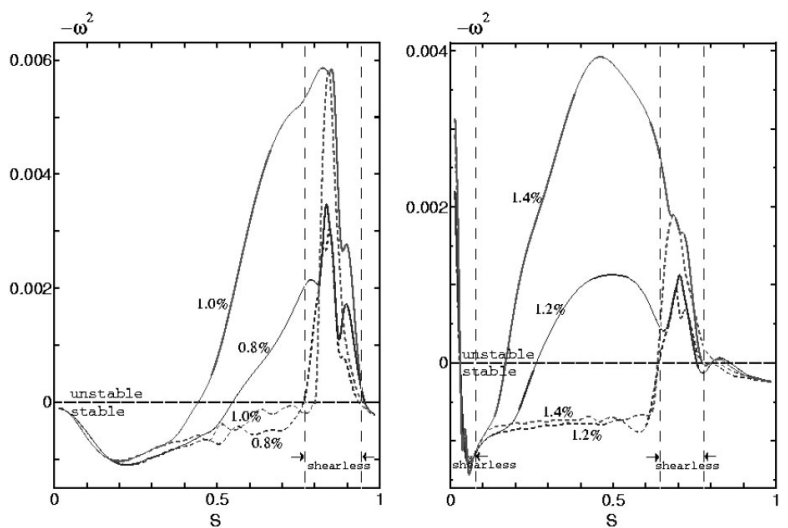

FIG. 9. Radial dependence of the eigenvalue for the broad (left) and peaked (right) pressure profile, as a function of $s$. The solid lines and the dashed lines are for $M \alpha=0$ and $M \alpha=\pi$ cases, respectively. Central beta values are affixed and the regions between dashed bar lines correspond to shearless regions.

structure reflects the strong toroidal mode coupling as $n$ increases. ${ }^{6}$ Since the toroidal mode coupling occurs through the $M$ number, which is large in LHD, the global mode corresponding to the local modes of the ballooning branch can have only a high- $n$ toroidal mode number, at least $n \gtrsim M$. On the other hand, the local modes of the interchange branch can reflect the low- $n$ global modes.

\section{B. Ballooning mode analysis in Heliotron-J}

Next the results for an $L=1 / M=4$ heliotron, Heliotron-J, are shown. In Fig. 9, the radial dependence of eigenvalues is shown for the broad pressure profile $p$ $=p(0)\left(1-s^{2}\right)^{2}$ and the peaked pressure profile $p=p(0)(1$ $-s)^{2}$ at several beta value near the marginal stability. The input $\theta_{k}$ is fixed to zero to take account of the modes in the most unfavorable region due to the toroidicity. In order to see the nonaxisymmetric effect, the $M \alpha$ is taken to be 0 and $\pi$, which correspond to the unfavorable and favorable curvature region due to the helicity. The spiky behavior of eigenvalue curves in the region between two dashed bar lines corresponds to the shearless region in the equilibrium as seen in Fig. 3(b). We must exclude these regions where the ballooning formalism is broken as mentioned in Sec. III. It can be seen that the modes for $M \alpha=0$ become unstable at relatively low beta, whereas for $M \alpha=\pi$ cases, the modes remain stable compared with $M \alpha=0$ cases except for the shearless region. The critical beta value for $M \alpha=0$ cases is $\beta_{0} \sim 0.7 \%$ for the broad pressure profile and $\beta_{0} \sim 1.1 \%$ for the peaked pressure profile. For $M \alpha=\pi$ cases, significant instabilities cannot be observed up to $\beta_{0}=2.0 \%$. Thus it turns out that $\alpha$ dependence of local ballooning modes is quite strong in Heliotron-J.

This is highlighted in Fig. 10, where the contours of unstable eigenvalue on $\theta_{k}-\alpha$ plane for fixed $s(=0.55)$ are shown. The stable eigenvalues are taken to be zero in this figure. It is found that the unstable eigenvalues are localized around $M \alpha=2 n \pi$ with any integer $n$, and the stable bands lie around $M \alpha=(2 n+1) \pi$ between unstable regions. Owing to the angle-like behavior or translational symmetry on

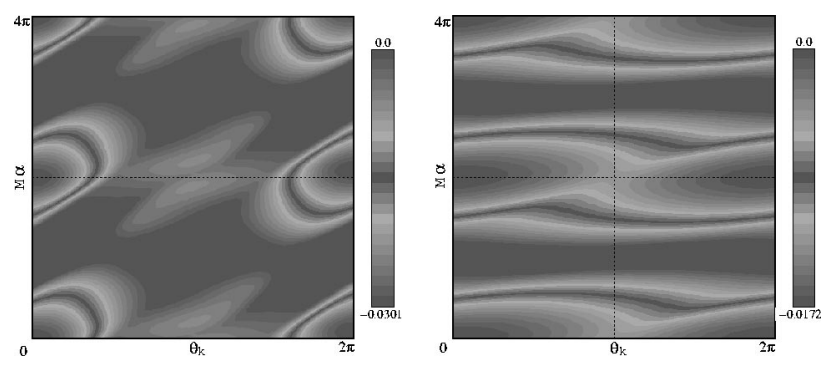

FIG. 10. Contours of the eigenvalues at $s=0.55$ for $\beta_{0}=2.0 \%$ Heliotron J equilibrium for the broad (left) and peaked (right) pressure profiles. The unstable modes are localized around $M \alpha=2 n \pi$ in the parameter space and the stable bands lies between the unstable regions.

$\theta_{k},{ }^{1}$ the eigenvalue isosurfaces form the isolated spheres around $\left(\theta_{k}, M \alpha\right) \sim(2 m \pi, 2(n-m M q) \pi)$ with $m, n=0$, \pm 1 , $\pm 2, \ldots$, in the parameter space $\left(s, \theta_{k}, \alpha\right)$. Therefore it is found again that the local modes have very strong $\alpha$ dependence, and there is no cylindrical isosurface of "interchange branch"' in Heliotron $\mathrm{J}$.

We now discuss the roots of instabilities and the dependence of the modes on the local parameter. As is well known, the ballooning stability is determined by the competition between the stabilizing field line tension and the destabilizing unfavorable magnetic curvature. Thus we need to explain the occurrence of unstable ballooning modes from the viewpoints of both the stabilizing and destabilizing effects. As seen from Eqs. (2) and (6), the stabilizing term $\mathcal{A}$ proportional to $\left|\mathbf{k} / k_{\alpha}\right|^{2}$ is expressed in terms of the product of integrated local shear along the field line and the measure of flux surface interval $|\nabla s|^{2}$, apart from the nonsecular term $B^{2} /|\nabla \chi|^{2}$. The $|\nabla s|^{2}$ is a function that becomes large outside and small inside the torus by the Shafranov shift. It is then obvious that the $\mathcal{A}$ increases as $\left|\theta-\theta_{k}\right|$ increases through the global shear part $|\nabla s|^{2}\left[\int s_{*} d \theta\right]^{2}$ or simply $|\nabla s|^{2} s_{*}^{2}(\theta$ $\left.-\theta_{k}\right)^{2}$ of it, and shows spikes due to the $|\nabla s|^{2}$ at $\theta \sim$ $\pm 2 \pi p$ with any natural number $p$ where the field line passes the outside of the torus. As shown in Ref. 11, however, the oscillations between negative and positive values of the integrated oscillating shear, the ripple average of which behaves odd-like in $\theta$ due to the covariant metric element $g_{s} \theta$, cancel the stabilizing effect of integrated global shear. That is, the cross term of the integrated local shear, $|\nabla s|^{2} \int s_{*} d \theta \int \widetilde{s}_{*} d \theta$, which is independent on $|\nabla s|^{2}$ as seen from Eq. (8), cancels the global shear part, and causes the reduction of stabilizing effect $\mathcal{A}$ after $\theta \sim \pm 2 \pi p$ along the field line. This reduction is more effective in the low shear system than in high shear system, since the global shear part can increase rapidly along the field line in the latter case. This is confirmed by comparing Fig. 11(a) with Fig. 11(b) where the associated quantities on the field line are shown. In Fig. 11(a) where the $q^{\prime}$ is small, the reduction of the integrated local shear can be seen as pointed by arrows, and the eigenfunction extended along the field line shows local maxima at these points. On the other hand, in the case of Fig. 11(b) with somewhat larger shear, the eigenfunction shows a more localized ballooning structure along the field line with more unstable eigenvalue, and the reduction of integrated local shear does not affect the mode. In this case, the stability 

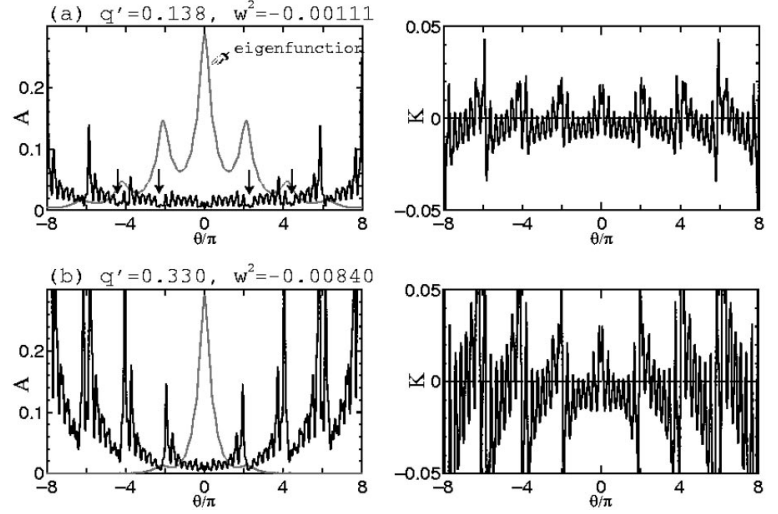

FIG. 11. Associated quantities along the field line; the stabilizing term $\mathcal{A}$ (left) and the possibly destabilizing term $\mathcal{K}$ (right) at $\beta_{0}=1.2 \%$. (a) $\left(s, \theta_{k}, M \alpha\right)=(0.5,0,0)$ for the peaked pressure profile. (b) $\left(s, \theta_{k}, M \alpha\right)$ $=(0.6,0,0)$ for the broad pressure profile. The $q^{\prime}$ values shown are proportional to global shear, $s_{*} \propto-q^{\prime}$. The reductions of the integrated local shear in the field line tension $\mathcal{A}$, pointed by arrows are realized more clearly in (a) with small global shear and the eigenfunction uprise there. In the case of (b) with larger shear, the mode structure is more localized.

of modes is mostly determined by the competition between the stabilizing and destabilizing effects near $\theta \sim \theta_{k}$.

As regarding the possibly destabilizing effect of $\mathcal{K}$ in Eq. (3), this arises mainly from the normal curvature, which is approximately the radial derivative of the magnetic field strength, $B^{-1} \partial B / \partial s$ at low beta considered here, as seen from Eq. (4). As shown in Fig. 2, the bumpy component $B_{0, M}$ and its radial derivative have an inverse sign to other dominant components, which is achieved by the control of the toroidal coil currents. ${ }^{7}$ Such a bumpy field can locally compensate the weak field due to the helicity $B_{L, M}$ and toroidicity $B_{1,0}$ at $(\theta, M \zeta) \sim(0,0)$, where $B(s, \theta, \zeta)$ $=\Sigma_{m, n} B_{m, n}(s) \cos (m \theta-n \zeta)$ and $(L, M)=(1,4)$ for Heliotron J. Although it contributes unfavorably against the favorable helicity at $(\theta, M \zeta) \sim(0, \pi)$, it is not strong enough to cancel the favorable helicity. The curvature is indeed favorable near $(\theta, M \zeta) \sim(0, \pi)$, as shown in Fig. 4(a). In addition, the favorable curvature region near $(\theta, M \zeta)=(\pi, 0)$ due to the helicity and toroidicity, is further improved by the bumpiness. Then the most favorable curvature area is formed there. Hence a field line passing near the point, $(\theta, M \zeta) \sim(0, \pi)$ is soaked in the deep well of good curvature and a mode with corresponding parameter, $M \alpha \sim(2 n+1) \pi$ is stabilized. This causes the strong $\alpha$ dependence of the local dispersion relation. From the viewpoint of the MHD stability, therefore, the bumpy field (and its radial derivative) is favorable against the ballooning modes so far as properly optimized. It is noted that the radial derivative of the bumpy field also plays an important role in the reduction of trapped particle losses due to the enhancement of poloidal drift. ${ }^{14}$

As already noted above, if a mode localization becomes stronger due to the increase of the global shear, the local reduction of stabilizing effect $\mathcal{A}$ near $\theta \sim \pm 2 \pi p$ is less important than for a more extended mode. This might be expected as a stabilizing effect of the global shear. However, comparing Fig. 11(a) with Fig. 11(b) shows that the mode is more unstable in the case with larger shear. This can be
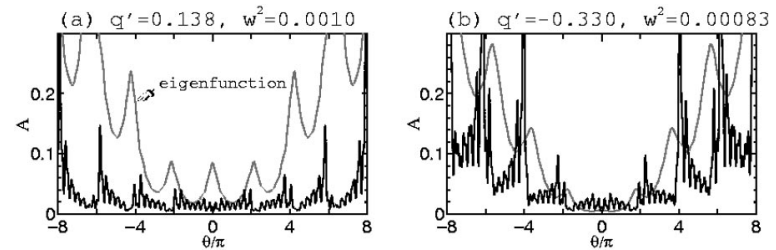

FIG. 12. Stabilizing term $\mathcal{A}$ with the same input $\left(s, \theta_{k}, M \alpha\right)=(0.6,0,0)$ for the broad pressure profile as in Fig. 11(b) except for $q^{\prime}$ changed artificially. (a) Taking $q^{\prime}=0.138$ which is the same value as in Fig. 11(a). (b) Taking $q^{\prime}=-0.330$ which is the inverse sign of that in Fig. 11(b).

understood by taking account of the stabilizing effect from the term $\mathcal{K}$, since a sufficiently localized mode cannot feel the stabilizing effect of negative $\mathcal{K}$ effectively. This is confirmed by comparing Fig. 11(b) with Fig. 12(a), where the stabilizing term $\mathcal{A}$ is shown for the same input parameters in Fig. 11(b) except for artificially changed $q^{\prime}$. As shown in Fig. 12(a) where $q^{\prime}$ is decreased to the same value as that in Fig. 11(a), the mode is stabilized by picking up the stabilizing $\mathcal{K}$ effects over long range, which is just the effect of magnetic well. These indicates that the mode in this low shear heliotron is near the second stability regime.

In Fig. 11(a), the unstable mode with small $q^{\prime}$ shows the extended structure along the field line. This is because all the secular terms in Eq. (1) are included as a combination with $q^{\prime}$. It is then obvious that the $\theta_{k}$ dependence becomes weak in low shear systems, and this explains the reason why the local dispersion relation shows the weak $\theta_{k}$ dependence in the case of the peaked pressure profile in Fig. 10 (right). In the global viewpoint, the perturbation, whose amplitude is maximum usually at the outside of torus, is twisted radially due to the global shear as flux tubes rotate poloidally, as shown in Fig. 13 of Ref. 6. As the global shear increases the mode is more localized poloidally, and finally would reach to the first stability regime. Otherwise, when the system has a small shear but is not in the second stability, the perturbation can rotate more easily along the flux tubes.

In addition to the magnitude of the global shear, the stellarator shear $\left(q^{\prime}<0\right)$ is often considered to be useful for stabilizing the ballooning modes. The reversed shear tokamak is such an example. ${ }^{15}$ As seen in Eqs. (3) and (6), the sign of the global shear affects the modes only through the geodesic curvature part of the term $\mathcal{K}$ and the cross term of the integrated local shear. When the global shear is stellarator-like, the geodesic curvature has certainly a stabilizing effect for modes concentrated in the outside of torus, because the $\kappa_{g}$ has an odd parity $\sim \sin \theta$ when helical ripples are averaged out. However, this effect does not dominate over the normal curvature part. As for the cross term of the integrated local shear, if we change the sign of $q^{\prime}$ artificially without changing any other local quantities, the mode is stabilized readily, as shown in Fig. 12(b). In this case, the reductions of integrated local shear occur at the inside of $\theta \sim$ $\pm 2 \pi p$ along the field line, since the cross term changes the phase hundred-and-eighty-degree through the change of the sign of $q^{\prime}$. Then the shape of $\mathcal{A}$ becomes cascade-like and the mode is stabilized due to the stabilizing $\mathcal{K}$ effects over long range. However, Nakajima ${ }^{11}$ pointed out that the phase 
of integrated oscillating shear nearly synchronizes with the sign of the global shear in the actual equilibrium. This indicates that it is invalid to change the sign of $q^{\prime}$ artificially without changing the phase of the integrated oscillating shear. After all, the stellarator shear $\left(q^{\prime}<0\right)$ affects the eigenvalue partly through the geodesic curvature, but we cannot expect that it can stabilize the nonaxisymmetric modes, unlike the case of the tokamak-like ballooning modes in such a reversed shear tokamak.

As a consequence of the strong $\alpha$ dependence in Heliotron $\mathrm{J}$, it is expected that the modes cannot spread over a flux surface, at least in the toroidal direction. This is consistent with the fact that the Mercier modes are stable in $\mathrm{He}$ liotron J equilibrium considered here, since the local ballooning modes corresponding to the Mercier modes should have the weak $\alpha$ dependence, on the analogy of the "interchange branch' in LHD. The quantization of such a mode for specific $n$ is impossible, since no cylindrical surface whose axis is in $\alpha$ direction exists. On the analogy of "ballooning branch" in LHD, the toroidal mode coupling should be strong in the global stability. However, it is expected that the local modes in Heliotron $\mathrm{J}$ can reflect the relatively low- $n$ global modes unlike those of the ballooning branch in LHD, because $M=4$ of Heliotron $\mathrm{J}$ is smaller than $M=10$ of LHD.

\section{CONCLUSIONS}

Ideal MHD stability analysis of the local ballooning modes in Heliotron $\mathrm{J}$ is investigated by means of the ballooning formalism and the results are compared with the LHD results. It is found in Heliotron $\mathrm{J}$ that the weak global shear makes the local modes unstable through the reduction of the integrated local shear. Such a mode tends to be extended along the field line, and the $\theta_{k}$ dependence of the modes becomes weak, while the strong bumpiness stabilizes the modes on the specific flux tubes passing through the favorable curvature region. As a result, the mode with weak $\alpha$ dependence cannot appear, and the local dispersion relation shows strong $\alpha$ dependence. We found that the nonaxisymmetric mode in Heliotron $\mathbf{J}$ equilibrium with the deep magnetic well can become stable if we decrease the global shear further. Therefore, it can be considered that the nonaxisymmetric mode in Heliotron $\mathbf{J}$ is near the second stability regime, by the similarity of the $\bar{s}$ - $\bar{\alpha}$ diagram in the tokamak. In the LHD equilibrium with the broad pressure profile, the interchange-like modes with weak $\alpha$ dependence become unstable first as beta increases, and it is found that such a mode appears in the Mercier unstable region. On the other hand, the nonaxisymmetric ballooning modes with strong $\alpha$ and $\theta_{k}$ dependence can be suppressed up to higher beta in favor of large global shear. We can consider that the nonaxisymmetric modes in LHD are in the first stability regime at low beta. Therefore, the local dispersion relation in an LHD equilibrium with the broad pressure profile shows the coexistence of the weak and strong $\alpha$ dependence, when nonaxisymmetric modes become unstable. From the comparison of such Mercier stable and unstable equilibria, we can conclude that an equilibrium in which only the mode with strong $\alpha$ dependence appears is Mercier stable.
The global shear is favorable for the stabilization of the nonaxisymmetric ballooning modes in the cases when it is sufficiently strong or weak. This feature is very similar to the ballooning modes in a tokamak, except for the fact that in the former case the sign of the global shear does not affect the local stability significantly. The bumpiness, which is important for the particle confinement as well as the Mercier stability, also turns out to be useful for the stabilization of the ballooning modes. One of the important properties of Heliotron $\mathbf{J}$ is the flexibility of the magnetic configurations so that proper optimization is possible. Two scenarios can be considered in order to stabilize the ballooning modes in Heliotron J. One is setting our sights on the first stability by changing the vacuum configuration to make the global shear strong at finite beta. This is actually possible and we have already confirmed the existence of an equilibrium stable up to the equilibrium beta limit, although we present here only the unstable cases for the nonaxisymmetric ballooning modes. The other is aiming at the second stability, which can be possible if the deep magnetic well is compatible with the sufficiently weak global shear at finite beta. This can be achieved if we can control the safety factor profile by the net current. More configuration studies are needed to clarify the stability properties of Heliotron $\mathrm{J}$ against the ballooning modes. Moreover, the fixed boundary constraint is used in the equilibrium calculation for simplicity in this study. It is desirable to calculate the equilibrium with free boundary in order to reproduce a realistic configuration in experiments. Since we have both the stable and unstable equilibrium configurations for the nonaxisymmetric ballooning mode at low beta, it will be very interesting if the existence of the nonaxisymmetric ballooning mode is demonstrated experimentally in near future. This will be reported in a different paper.

It is well known that the kinetic effects, such as finite Larmor radius, give rise to stabilizing effects for strongly localized shear-Alfvén modes, as well as drift modes. The kinetic analysis is needed to determine the actual stability in Heliotron J. In addition, since there is no isosurface whose axis is in $\alpha$ direction in Heliotron $\mathrm{J}$, we cannot use the quantization condition to estimate finite- $n$ results. Particularly for the case with very weak $\theta_{k}$ dependence and quite strong $\alpha$ dependence in such a peaked pressure profile case, it is difficult to predict the appearance of the global modes from the local modes, because this requires one to superimpose the infinitely degenerated $\theta_{k}$ branches in the local analysis. The global analysis must be performed directly to investigate the stability of the finite- $n$ modes.

\section{ACKNOWLEDGMENTS}

The authors thank Dr. N. Nakajima for fruitful discussions. Also the authors are grateful to Professor M. Wakatani for helpful suggestions.

\footnotetext{
${ }^{1}$ R. L. Dewar and A. H. Glasser, Phys. Fluids 26, 3038 (1983).

${ }^{2}$ N. Nakajima, Phys. Plasmas 3, 4556 (1996).

${ }^{3}$ W. A. Cooper, D. B. Singleton, and R. L. Dewar, Phys. Plasmas 3, 275 (1996).

${ }^{4}$ P. Cuthbert, J. L. V. Lewandowski, H. J. Gardner, M. Persson, D. B.
} 
Singleton, R. L. Dewar, N. Nakajima, and W. A. Cooper, Phys. Plasmas 5, 2921 (1998).

${ }^{5}$ Y. Nakamura, T. Matsumoto, M. Wakatani et al., J. Comput. Phys. 128, 43 (1996).

${ }^{6}$ J. Chen, N. Nakajima, and M. Okamoto, Phys. Plasmas 6, 1562 (1999).

${ }^{7}$ M. Wakatani, Y. Nakamura, K. Kondo, M. Nagasaki, S. Besshou, T. Obiki, F. Sano, K. Hanatani, T. Mizuuchi, H. Okada, and M. Yokoyama, Nucl. Fusion 40, 569 (2000).

${ }^{8}$ S. P. Hirshman, Phys. Fluids 26, 3553 (1983).

${ }^{9}$ Y. Nakamura, M. Wakatani, and K. Ichiguchi, J. Plasma Fusion Res. 69, 41 (1993).

${ }^{10}$ W. D. D'haeseleer, W. N. G. Hitchon, J. D. Callen, and J. L. Shohet, Flux
Coordinates and Magnetic Field Structure (Springer-Verlag, Berlin, 1991).

${ }^{11}$ N. Nakajima, Phys. Plasmas 3, 4545 (1996).

${ }^{12}$ J. P. Freidberg, Ideal Magnetohydrodynamics (Plenum, New York, 1987).

${ }^{13}$ L. F. Shampine and M. K. Gordon, Computer Solution of Ordinary Differential Equations: The Initial Value Problem (Freeman, San Francisco, 1975).

${ }^{14}$ M. Yokoyama, N. Nakajima, M. Okamoto, Y. Nakamura, and M. Wakatani, Nucl. Fusion 40, 261 (2000).

${ }^{15}$ M. W. Phillips, M. C. Zarnstorff, J. Manickam, F. M. Levinton, and M. H. Hughes, Phys. Plasmas 3, 1673 (1996). 